\title{
Calculation of Boron Nitride Sputter Yields Under Low Energy Xenon Ion Bombardment
}

\author{
John T. Yim, Michael Falk $†$ Michael Keidar; and Iain D. Boyd ${ }^{\S}$ \\ University of Michigan, Ann Arbor, MI 48109, USA
}

\begin{abstract}
An accurate description of the sputter yield of boron nitride (BN) from xenon ion bombardment at low energies is needed for improving the prediction capabilities of Hall thruster erosion models and in turn for lifetime prediction models. However, sputter yield data at low $(<300 \mathrm{eV})$ energies does not exist. The molecular dynamics method is employed to model the sputtering of $\mathrm{BN}$ at low energies. The results are compared to existing experimental data at higher energies. A qualitative comparison with a quantumstatistical model is also performed.
\end{abstract}

\section{Introduction}

$\mathrm{O}$ $\mathrm{NE}$ of the main failure mechanisms for Hall thrusters is the erosion of the acceleration channel walls. Recently, there has been work to model this erosion. ${ }^{1-4}$ Simulation model development is important for thruster validation since full lifetime testing involves a prohibitive amount of time and money. These models usually take the form of calculating the ion fluxes to the walls and using these as input to a semi-empirical sputter yield model to compute wall erosion. ${ }^{5,6}$ The sputter yield model is often a curve fit to published experimental data of boron nitride sputter yields. ${ }^{7}$ However, experimental data do not exist for ion energies below $300 \mathrm{eV}$ and thus the very low energy portions of the sputter yield curve fits need to be extrapolated from existing data. Unfortunately, the predicted erosion rate of thruster channel walls has been shown to be highly affected by the sputter yields in this low energy region. ${ }^{8}$ This is especially of importance to Hall thruster erosion evaluations for thrusters that have an overall potential drop in the hundreds of volts since the ions impacting the walls will be in this low energy regime.

In the present work, a molecular dynamics method is chosen to evaluate the sputter yields at the very low energies. Experimental procedures can be difficult to perform accurately as the energies approach the threshold value required for sputtering. Monte-Carlo methods were also considered as they have been used for sputter analyses before. ${ }^{9-12}$ However, again, the low energies under consideration for this study introduce uncertainties of the underlying assumptions of such methods, including binary collisions. Thus, though the molecular dynamics method may be more computationally intensive than Monte-Carlo methods, it is expected that the results would be more accurate. An overview of the molecular dynamics model is described in the next section. The subsequent section will provide results from the model and comparisons to experimental data, followed by conclusions and areas of future improvement.

\section{Method}

The molecular dynamics method solves Newton's law of motion for a system of particles, which in this case are atoms. The interactions among the particles are determined through interatomic potential functions. Since only conservative forces are considered, the negative gradient of the potential function provides the forces on each particle arising from the particles around it. The traditional leapfrog method is used to calculate the velocities halfway in between each timestep, when forces and positions are calculated.

\footnotetext{
${ }^{*}$ Graduate Student, Department of Aerospace Engineering, AIAA Student Member.

$\dagger$ Associate Professor, Department of Materials Science and Engineering.

¥Assistant Research Scientist, Department of Aerospace Engineering, AIAA Senior Member.

$\S$ Professor, Department of Aerospace Engineering, AIAA Associate Fellow.
} 
Averaging over a sufficient number of timesteps and events, the macroscopic quantities of interest, here specifically, the sputter yield, can be obtained. Molecular dynamics is a valid tool for this purpose and has been used in other sputter analysis studies. ${ }^{13-16}$

For the interactions of the boron and nitrogen atoms with one another, the interatomic potential presented by Albe et al is used. ${ }^{17,18}$ This is a modified form of the multi-body potential first proposed by Tersoff for multi-component systems. ${ }^{19}$ The Tersoff potential has been used to model other covalent systems successfully and the general form is well suited for the purposes here. In particular, Albe et al have used their potential to model hexagonal boron nitride, which is the same form that is modeled here. ${ }^{17}$ They achieve good agreement with values of structural and elastic properties, such as the bulk modulus and lattice constant, recorded from other methods including experimental data.

The potential is of the form

$$
\Phi=\frac{1}{2} \sum_{i \neq j} f_{c}\left(r_{i j}\right)\left[f_{R}\left(r_{i j}\right)-b_{i j} f_{A}\left(r_{i j}\right)\right]
$$

where $f_{c}$ is a cutoff function limiting the range of the potential. The $f_{R}$ and $f_{A}$ terms are the repulsive and attractive parts of the potential, respectively. The $b_{i j}$ term is a modifier to the attractive force component that takes into account third-body bond stretching and bending, and $r_{i j}$ is the distance from particle $i$ to particle $j$. The potential is dependent on particle positions only.

The repulsive and attractive components to this potential are similar in form and are based off of a Morse potential $^{20}$

$$
\begin{aligned}
& f_{R}\left(r_{i j}\right)=\frac{D_{0}}{S-1} \exp \left[-\beta \sqrt{2 S}\left(r_{i j}-r_{0}\right)\right] \\
& f_{A}\left(r_{i j}\right)=\frac{S D_{0}}{S-1} \exp \left[-\beta \sqrt{\frac{2}{S}}\left(r_{i j}-r_{0}\right)\right]
\end{aligned}
$$

where $D_{0}$ is the dimer energy and $r_{0}$ is the dimer separation. $S$ and $\beta$ are fitting constants.

The modifier term $b_{i j}$ is given as

$$
\begin{gathered}
b_{i j}=\left(1+\gamma^{n} \chi^{n}\right)^{-1 / 2 n} \\
\chi=\sum_{k \neq i, j} f_{c}\left(r_{i k}\right) g\left(\theta_{i j k}\right) h\left(r_{i j}, r_{i k}\right) \\
g\left(\theta_{i j k}\right)=1+\frac{c^{2}}{d^{2}}-\frac{c^{2}}{d^{2}+\left(m-\cos \left(\theta_{i j k}\right)^{2}\right.} \\
h\left(r_{i j}, r_{i k}\right)=\exp \left[\lambda^{3}\left(r_{i j}-r_{i k}\right)^{3}\right]
\end{gathered}
$$

where the $k$ index indicates the third particle under consideration $\left(\theta_{i j k}\right.$ would be the angle between $i j$ and $i k)$ and $\gamma, n, c, d, m$, and $\lambda$ are all constants.

The cutoff function, $f_{c}$, is changed from the one used by Albe et al. ${ }^{17}$ Originally, it was of the form

$$
f_{c}\left(r_{i j}\right)= \begin{cases}1, & r_{i j} \leq R-D \\ \frac{1}{2}-\frac{1}{2} \sin \left[\pi\left(r_{i j}-R\right) /(2 D)\right], & R-D<r_{i j}<R+D \\ 0, & r_{i j} \geq R+D\end{cases}
$$

where $D$ and $R$ set the cutoff range of the potential. Though this cutoff potential transitions smoothly from unity to zero and its first derivative also goes to zero at the boundaries (at $R-D$ and $R+D$ ), the second derivative is not zero at the boundaries. This results in a small 'hiccup' in the total energy whenever bonds are broken or made. To resolve this issue, the cutoff function was changed to ${ }^{21}$

$$
\begin{gathered}
f_{c}\left(r_{i j}\right)= \begin{cases}1, & r_{i j} \leq R-D \\
\exp \left(\alpha \frac{x^{3}}{x^{3}-1}\right), & R-D<r_{i j}<R+D \\
0, & r_{i j} \geq R+D\end{cases} \\
\quad x=\frac{r_{i j}-(R-D)}{2 D}
\end{gathered}
$$


where $\alpha=3$ minimizes the magnitude of the local extremum of the first derivative. This function transitions smoothly from unity to zero while both the first and second derivatives are zero at the boundaries. The first derivative-which corresponds to the force resulting from the potential-is shown for both cutoff functions in Fig. 1.

An additional modification is made to the potential. Initial runs using the Albe potential did not conserve energy successfully. The first reason is due to the cutoff potential, as described above. The second arises from the $g$ component within the modifier $b_{i j}$ in Eq. (6). The coefficients $c$ and $d$ are adjusted from 0.52629 and 0.001587 to 3.316257 and 0.01 respectively, since the low value of $d$ makes the third term in Eq. (6) very sensitive to the angle $\theta_{i j k}$. This is shown in Fig. 2. As can be seen, the original coefficients create a very steep profile that would require a much smaller timestep to traverse smoothly. The value $c$ is also altered to keep the second term of $g$ given in Eq. (6) the same value as it was originally. Table 1 lists the constants used for this potential and their value depending on the identity of the pair $i$ and $j$.

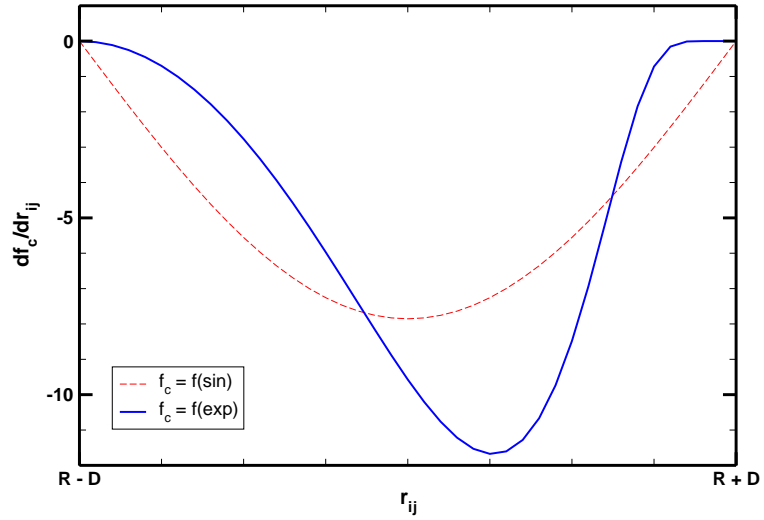

Figure 1. The first derivative of the cutoff functions given in Eq. (8) (sin) and Eq. (9) (exp).

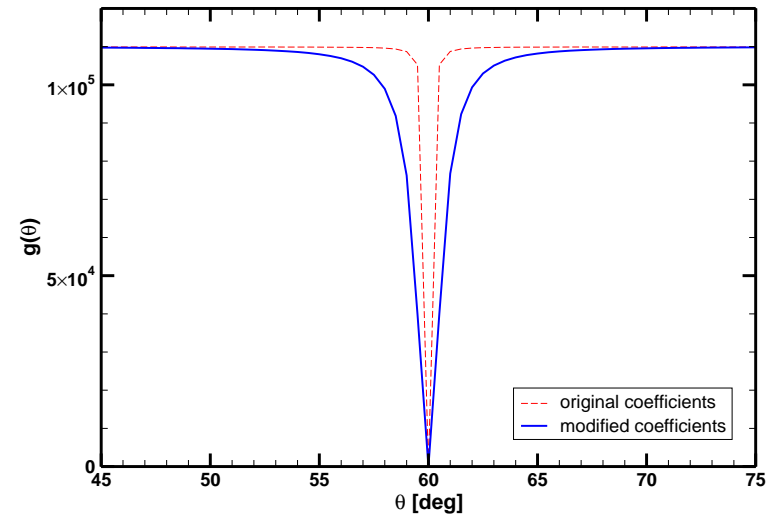

Figure 2. The $g$ term in Eq. (6) for B-B bonds using the original and modified coefficients.

\begin{tabular}{llll}
\hline \hline & B-B & N-N & B-N \\
\hline$R[\AA]$ & 2.0 & 2.0 & 2.0 \\
$D[\AA]$ & 0.1 & 0.1 & 0.1 \\
$D_{0}[\mathrm{eV}]$ & 3.08 & 9.91 & 6.36 \\
$r_{0}[\AA]$ & 1.59 & 1.11 & 1.33 \\
$\beta\left[\AA^{-1}\right]$ & 1.5244506 & 1.92787 & 2.043057 \\
$S$ & 1.0769 & 1.0769 & 1.0769 \\
$n$ & 3.9929061 & 0.6184432 & 0.364153367 \\
$\gamma$ & 0.0000016 & 0.019251 & 0.000011134 \\
$\lambda\left[\AA^{-1}\right]$ & 0.0 & 0.0 & 1.9925 \\
$c$ & 3.316257 & 17.7959 & 1092.9287 \\
$d$ & 0.01 & 5.9484 & 12.38 \\
$m$ & 0.5 & 0.0 & -0.5413 \\
\hline \hline
\end{tabular}

Table 1. Coefficient values used for the boron nitride potential function.

For the interactions involving the xenon ions, a Molière potential is used. The Molière potential is a purely repulsive potential. Since the van der Waals attraction of the xenon with the boron and nitrogen atoms is much weaker than the covalent attraction the boron nitride has within itself, a purely repulsive force is acceptable. ${ }^{22}$ This potential is based on a screened Coulomb repulsion and has the form

$$
\Phi=\frac{Z_{i} Z_{j} e^{2}}{4 \pi \epsilon_{0} r_{i j}}\left[0.35 \exp \left(-0.3 \frac{r_{i j}}{a_{F}}\right)+0.55 \exp \left(-1.2 \frac{r_{i j}}{a_{F}}\right)+0.10 \exp \left(-6.0 \frac{r_{i j}}{a_{F}}\right)\right]
$$

where $a_{F}$ is the Firsov screening length. 
The boron nitride considered here is in hexagonal form, similar in structure to that of graphite. The domain consists of twelve sheets of $9 \times 12$ hexagons, or 5400 particles arranged in a $3.9 \mathrm{~nm} \times 3.9 \mathrm{~nm} \times 3.0 \mathrm{~nm}$ box. An end-on view is shown in Fig. 3. This and subsequent molecular visualizations are produced using the VMD software package. ${ }^{23}$ Periodic boundary conditions are applied in the lateral directions. The bottom layer of atoms is kept immobile to keep the structure as a whole from translating due to ion impacts. The next two layers directly above it are designated as a thermostatted region. The two thermostatted layers are regulated using a Berendsen thermostat which rescales the velocities of the particles involved. ${ }^{22,24}$ The rest of the boron nitride is regulated through conduction. The boron nitride is initialized at a temperature of $850 \mathrm{~K}$, which is approximately the temperature of the Hall thruster channel walls while in operation. ${ }^{4}$ This temperature is set through the initial velocities, which are randomized in such a way as to remove any bulk motion.

The temperature of the $\mathrm{BN}$ block is monitored using a sub-relaxation evaluation of the macroscopic temperature. ${ }^{25}$ Since the instantaneous temperature of the block incurs large statistical scatter, a way to evaluate the average temperature of the block is needed. An example of the temperature of the BN block involving an ion impact is shown in Fig. 4.

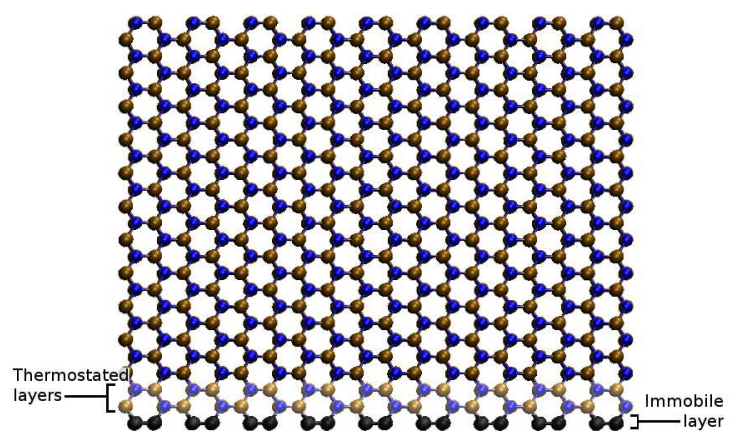

Figure 3. An end-on view of the boron nitride block showing the hexagonal structure and layers treated in the simulation.

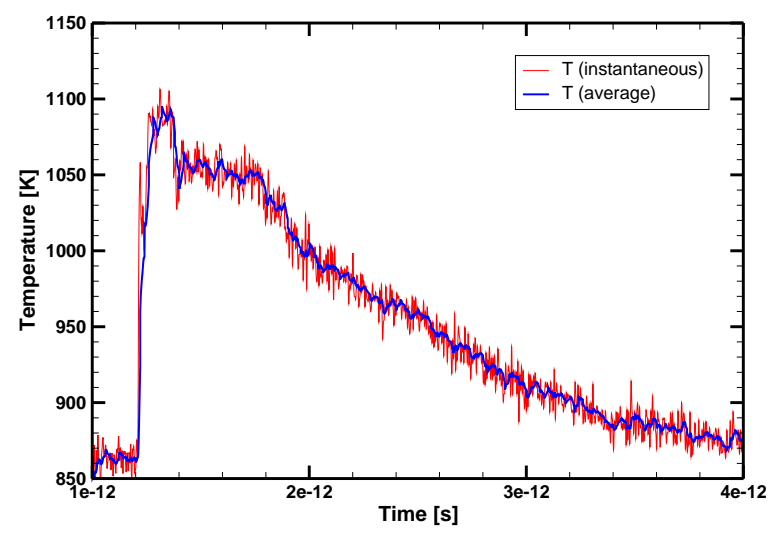

Figure 4. The instantaneous and average temperature as calculated using the sub-relaxation method.

After the boron nitride surface has equilibrated, a xenon ion is injected far above the surface with a specified energy. The angle incident to the surface normal is set to 60 degrees, as this is around where maximum sputtering occurs. ${ }^{7,26}$ The other angular component is randomized to minimize the effects of lattice orientation in the lateral directions. After a sufficient number of ion impact events, however, the upper regions of boron nitride form an amorphous layer that contains no observable orientation for the structure as seen in Fig. 5. After each ion impact, the boron nitride is re-equilibrated to the proper temperature before proceeding with the next ion impact.

In order to speed up the calculations, several computational techniques are employed. Neighbor lists, both Verlet neighbor and linked cell lists, are used so that each possible particle pair did not need to be evaluated at each timestep. Especially for a short range potential, such as the modified Tersoff described above, this removes the need to perform $\mathrm{O}\left(\mathrm{N}^{2}\right)$ calculations for every timestep. The simulation code is also parallelized with OpenMP directives for use on multi-processor machines. A timestep of $0.02 \mathrm{fs}$ is used to run the simulation.

The simulation is first run for a number of ion impacts until a representative form of boron nitride is formed with the amorphous layers near the surface. This state is then used as the initial block to test a sufficient number of individual ion impact events. The ion initial positions and velocities are randomized, but the energy and incident angle are kept the same. From these impact events, the statistics are gathered and analyzed to calculate the sputter yields. The properties of the ejected atoms are also kept track of to produce differential sputtering data. The ion energies examined varies from $350 \mathrm{eV}$, in order to compare to experimental data, to $50 \mathrm{eV}$, which is near where the sputter threshold energy for this system is expected to lie.

A quantum-statistical approach presented by Wilhelm is also used for comparison of the sputter yields at very low energies. ${ }^{27}$ A three-body interaction of the ion and two atoms of a polycrystalline solid is considered, and the probabilities of the ion scattering versus an atom sputtering are used to calculate the 


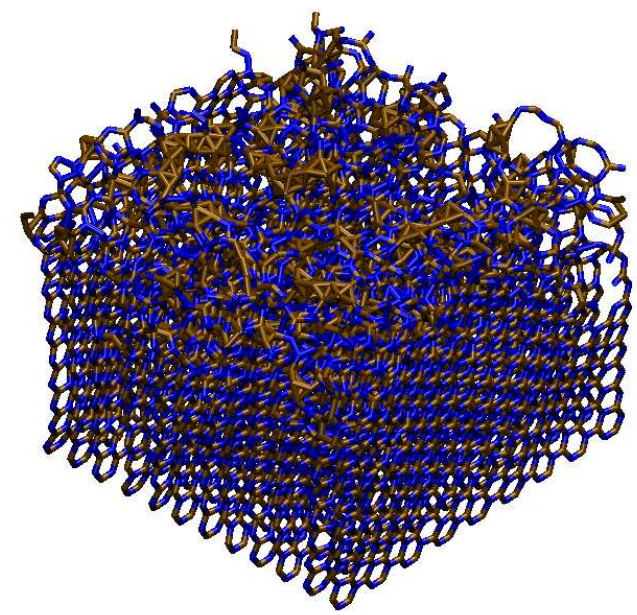

Figure 5. A view of a sample boron nitride surface (bonds only) after a number of xenon ion impacts.

rate of sputter. Wilhelm's approximation for the sputter yield at energies between the threshold energy and about $100 \mathrm{eV}$ is given by

$$
S(E) \approx \frac{1}{24} h_{2 / 1} \sigma\left(E_{0}\right) N^{2 / 3}\left(\frac{(M / m)^{2}}{1+2 M / m}\right)^{3 / 2} \frac{\left(E-E_{0}\right)^{2}}{E_{0}^{2}}
$$

where $S$ is the sputter yield, $E$ and $E_{0}$ are the energy and threshold energy respectively, $h_{2 / 1}$ is a dimensionless coefficient dependent on the perturbation operator of the Hamiltonian of the system between the initial and final states and their corresponding volumes, $\sigma$ is the total scattering cross section, $N$ is the number density of the atoms in the solid, and $m$ and $M$ are the mass of the ion and the target atom respectively. Since all of the parameters required for Eq. (12) are not known, only a qualitative form of the equation is used in this work for comparative purposes. Equation (12) is proportional to

$$
S(E) \approx a\left(E-E_{0}\right)^{2}
$$

where $a$ is a fitting coefficient.

\section{Results and Discussion}

The sputter yields are calculated for xenon ions impacting at an incidence angle of 60 degrees from the surface normal and initial energies of 350, 250, 150, 100, and $50 \mathrm{eV}$ and are shown in Fig. 6. Also shown are experimental data. ${ }^{7,26}$ For each of the calculated sputter yields, at least 100 ion impacts were simulated. The calculated sputter yield at $350 \mathrm{eV}$ is more than five times higher than measured experimentally. The results also show sputtering still occurring at $50 \mathrm{eV}$, which is at the low end of some estimates of the threshold energy. Thus, this simulation is predicting a higher sputter yield than expected overall. At this point, it is uncertain what exactly is causing the calculated sputter yield to be so much higher than the experimental values. One factor that is contributing to a higher sputter yield than expected is the temperature of the surface. The simulation is run with a base temperature of $850 \mathrm{~K}$ so as to simulate the working conditions inside a Hall thruster channel, which is the motivation and one end application behind this work. However, Garnier et al report temperatures of up to only $423 \mathrm{~K}$ when their data was collected. ${ }^{7}$ Also, as seen in Fig. 4 , an ion impact can raise the local temperature of the surface significantly, thus the base temperature of the solid may need to be kept even lower. Since lower temperature atoms will have less energy, lower amounts of sputtering would be expected.

Another anomaly encountered from this work is that nitrogen sputtered from the surface in much greater numbers than did boron. At $350 \mathrm{eV}$, the nitrogen sputtered more than three times the amount number-wise than boron. At $50 \mathrm{eV}$, only nitrogen is observed to sputter, albeit in smaller amounts than seen in the $350 \mathrm{eV}$ case, but no boron left the surface. An electron spectroscopy for chemical analysis (ESCA) study performed 


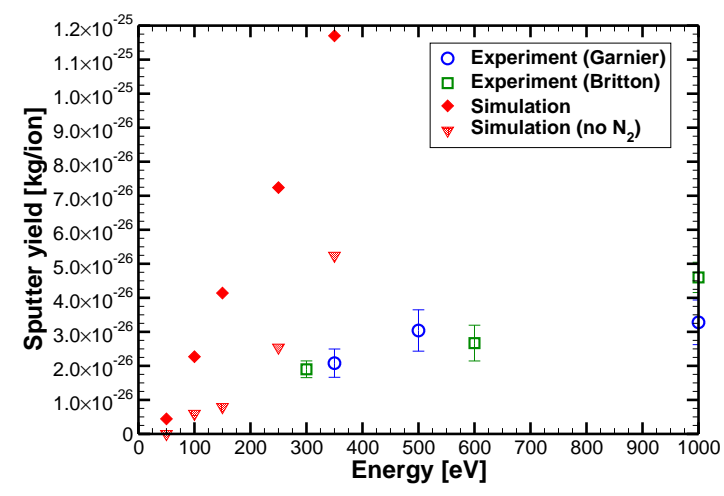

Figure 6. The sputter yield versus ion energy.

on a boron nitride surface after sputtering shows little variation between the boron and nitrogen ratios before and after sputtering. ${ }^{7}$ This indicates that there is no experimentally observed significant preferential sputtering of one element over the other.

Associated with the abundant nitrogen sputtering is how the atoms sputter from the surface. Table 2 shows how many times a particular atom or molecule is sputtered from the surface over 100 sample ion impacts for xenon ions at $350 \mathrm{eV}$. There is a preponderance of $\mathrm{N}_{2}$ molecules being sputtered from the surface, while there is a dearth of boron molecules being sputtered. This disparity mirrors the large proportion of nitrogen being sputtered compared to boron. A visual examination of the surface as in Fig. 5 shows the boron (shown in brown, nitrogen is blue) clumped together in groups whereas the nitrogen is more equally spread out over the surface. This affinity of the boron-boron bonds seems to cause the boron atoms to adhere more to the surface, and when they do sputter as molecules they have been observed in this model to sputter as $\mathrm{B}_{4}, \mathrm{~B}_{5}$, and $\mathrm{B}_{6}$ molecules, further showing their tendency to stay together. In particular, the nitrogen molecule appears to sputter much more readily than expected. At $50 \mathrm{eV}$, only nitrogen molecules are observed to sputter from the surface while no other compounds are ejected. This would seem to indicate a bond-order effect in the potential function where a nitrogen-nitrogen interaction saturates their bonding, making it more difficult for other bonds to form. The sputter yields are calculated ignoring the contribution of the $\mathrm{N}_{2}$ molecules, as shown in Fig. 6. The results are significantly closer to experimental data, though they are still greater than the experimental sputter yield around $300 \mathrm{eV}$ and above, signifying that the unbalanced proportion of $\mathrm{N}_{2}$ sputtering is not the sole cause of the sputter yield overestimation.

\begin{tabular}{cc|cc}
\hline \hline Compound & Number sputtered & Compound & Number sputtered \\
\hline $\mathrm{B}$ & 46 & $\mathrm{~N}$ & 64 \\
$\mathrm{~B}_{2}$ & 3 & $\mathrm{~N}_{2}$ & 140 \\
$\mathrm{~B}_{3}$ & 1 & $\mathrm{~N}_{3}$ & 1 \\
$\mathrm{~B}_{4}$ & 1 & $\mathrm{BN}$ & 42 \\
$\mathrm{~B}_{5}$ & 3 & $\mathrm{BN}_{2}$ & 7 \\
$\mathrm{~B}_{6}$ & 1 & $\mathrm{~B}_{2} \mathrm{~N}$ & 1 \\
\hline \hline
\end{tabular}

Table 2. Sputtered components from 100 ion impacts at $350 \mathrm{eV}$.

The results are also compared qualitatively to predictions from a quantum-statistical analysis of low energy sputtering. In general, the sputter yield at very low energies is predicted to be proportional to the square of the energy above the threshold value as seen in Eq. (13). Since there are two variables in the equation which are unknown-the threshold energy and the proportionality coefficient-the curve was matched to the two data points at 50 and $100 \mathrm{eV}$. Then, the corresponding threshold energy, $E_{0}$, is $11.4 \mathrm{eV}$ while $a=1.3 \times 10^{-4}$. Figure 7 shows this fit of the quantum-statistical trend against the results of the model. More data points are needed to better compare the two predictions of the sputter yield, but the threshold energy necessary to fit the first two points is much lower than the range the threshold energy is expected to lie in. This may have the same reasons behind it as explained above, namely the extra sputtered nitrogen 
are skewing the results. Other possible causes may be from the assumptions made in the quantum-statistical analysis. The analysis is originally performed for polycrystalline monatomic metals as opposed to graphitelike sheets of a binary compound. Also, the analysis is only applicable to very low energies, less than $100 \mathrm{eV}$ even.

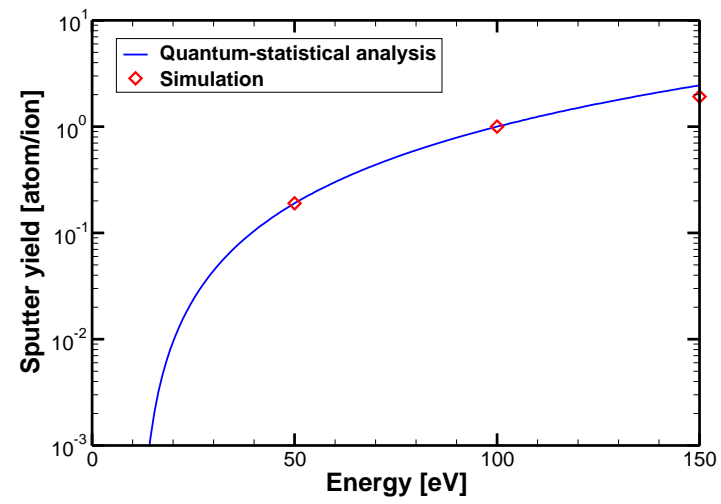

Figure 7. A fit from quantum-statistical analysis to the results of the model.

\section{Conclusions}

A molecular dynamics simulation is used to model the sputtering of hexagonal boron nitride from low energy xenon ion impacts. The sputter yields calculated are higher than expected, especially noticeable around $350 \mathrm{eV}$, where experimental data are available for comparison. The reason behind this result is not clearly understood, though there may be several factors at work. The temperature simulated is higher than the temperature measured when the experimental data were taken. Larger statistical sets are needed to confirm the results as being accurate representations of the macroscopic values. Only one crystal orientation was tested; other configurations may result in lower sputter yields, reducing the overall average.

A greater proportion of nitrogen, especially in the form of $\mathrm{N}_{2}$ molecules, is found to sputter from the surface in this simulation. There is no experimentally observed change in the element composition ratios before and after sputtering. The simulation is either adding a bond-order effect of N-N bonds that is not physically present, or it is neglecting other effects such as charge transfers between boron and nitrogen atoms and the consequent electrostatic interactions that may lead to higher nitrogen retention. Taking all of the above issues in account, further analyses using the molecular dynamics method may result in additional understanding of the BN sputtering process. Along with improved knowledge of the sputter yield at low energies, other investigations such as differential sputter analysis can be performed.

\section{Acknowledgments}

We would like to thank W.-K. Kim of the Department of Materials Science and Engineering at the University of Michigan for his help in implementing the potential functions.

Part of this work was supported by NASA Glenn Research Center through Grant NNCO4GA56G. The first author would also like to acknowledge support from the National Science Foundation through their Graduate Research Fellowship Program.

\section{References}

${ }^{1}$ Yu, D., Li, Y., and Song, S., "Ion Sputtering Erosion of Channel Wall Corners in Hall Thrusters," Journal of Physics D: Applied Physics, Vol. 39, 2006, pp. 2205-2211.

${ }^{2}$ Sommier, E., Allis, M. K., Gascon, N., and Cappelli, M. A., "Wall Erosion in 2D Hall Thruster Simulations," 42nd AIAA/SAE/ASME/ASEE Joint Propulsion Conference and Exhibit, July 2006, AIAA-2006-4656.

${ }^{3}$ Lovtsov, A., Shagayda, A., and Gorshkov, O., "Semi-Empirical Method of Hall Thrusters Lifetime Prediction," 42nd AIAA/ASME/SAE/ASEE Joint Propulsion Conference and Exhibit, July 2006, AIAA-2006-4661.

${ }^{4}$ Hofer, R. R., Katz, I., Mikellides, I. G., and Gamero-Castaño, M., "Heavy Particle Velocity and Electron Mobility 
Modeling in Hybrid-PIC Hall Thruster Simulations," 42nd AIAA/SAE/ASME/ASEE Joint Propulsion Conference and Exhibit, July 2006, AIAA-2006-4658.

${ }^{5}$ Manzella, D. H., Yim, J. T., and Boyd, I. D., "Predicting Hall Thruster Operational Lifetime," 40th AIAA/SAE/ASME/ASEE Joint Propulsion Conference and Exhibit, July 2004, AIAA 2004-3953.

${ }^{6}$ Yim, J. T., Keidar, M., and Boyd, I. D., "A Hydrodynamic-Based Erosion Model for Hall Thrusters," 29th International Electric Propulsion Conference, Oct. 2005, IEPC-2005-013.

${ }^{7}$ Garnier, Y., Viel, V., Roussel, J.-F., and Bernard, J., "Low-Energy Xenon Ion Sputtering of Ceramics Investigated for Stationary Plasma Thrusters," Journal of Vacuum Science and Technology A, Vol. 17, No. 6, Nov/Dec 1999, pp. 3246-3254.

${ }^{8}$ Yim, J. T., Keidar, M., and Boyd, I. D., "An Investigation of Factors Involved in Hall Thruster Wall Erosion Modeling," 42nd AIAA/SAE/ASME/ASEE Joint Propulsion Conference and Exhibit, July 2006, AIAA 2006-4657.

${ }^{9}$ Pugacheva, T. S., Jurabekova, F. G., and Valiev, S. K., "Effects of Cascade Mixing, Sputtering and Diffusion by High Dose Light Ion Irradiation of Boron Nitride," Nuclear Instruments and Methods in Physics Research B, Vol. 141, 1998, pp. 99-104.

${ }^{10}$ Chen, M., Rohrbach, G., Neuffer, A., Barth, K.-L., and Lunk, A., "Simulation of Boron Nitride Sputtering Process and Its Comparison with Experimental Data," IEEE Transactions on Plasma Science, Vol. 26, No. 6, Dec. 1998, pp. $1713-1717$.

${ }^{11}$ Greene, J. P., Nemanich, J., Thomas, G. E., and Schiel, S. L., "Noble Gas Sputtering Calculations Using TRIM," Nuclear Instruments and Methods in Physics Research A, Vol. 397, 1997, pp. 91-98.

${ }^{12}$ Eckstein, W. and Biersack, J. P., "Computer Simulation of Two-Component Target Sputtering," Applied Physics A, Vol. 37, 1985, pp. 95-108.

${ }^{13}$ Eltekov, V. A., Elovikov, S. S., Colligon, J. S., Negrebetskaya, N. N., Promokhov, A. A., and Yurasova, V. E., "Sputtering of Graphite-Like BN Crystals," Radiation Effects and Defects in Solids, Vol. 133, 1995, pp. 107-120.

${ }^{14}$ Promokhov, A. A., Mosunov, A. S., Elovikov, S. S., and Yurasova, V. E., "Features of Sputtering of Nitrides with Various Component Mass Ratios," Vacuum, Vol. 56, 2000, pp. 247-252.

${ }^{15}$ Zhou, X. W., Wadley, H. N. G., and Sainathan, S., "Low Energy Sputtering of Nickel by Normally Incident Xenon Ions," Nuclear Instruments and Methods in Physics Research B, Vol. 234, 2005, pp. 441-457.

${ }^{16}$ Kress, J. D., Hanson, D. E., Voter, A. F., Liu, C. L., Liu, X.-Y., and Coronell, D. G., "Molecular Dynamics Simulation of $\mathrm{Cu}$ and $\mathrm{Ar}$ Ion Sputtering of $\mathrm{Cu}$ (111) surfaces," Journal of Vacuum Science and Technology A, Vol. 17, No. 5, 1999, pp. 2819-2825.

${ }^{17}$ Albe, K., Möller, W., and Heinig, K.-H., "Computer Simulation and Boron Nitride," Radiation Effects and Defects in Solids, Vol. 141, 1997, pp. 85-97.

${ }^{18}$ Albe, K. and Möller, W., "Modelling of Boron Nitride: Atomic Scale Simulations on Thin Film Growth," Computational Materials Science, Vol. 10, 1998, pp. 111-115.

${ }^{19}$ Tersoff, J., "New Empirical Approach for the Structure and Energy of Covalent Systems," Physical Review B, Vol. 37, No. 12, April 1988, pp. 6991-7000.

${ }^{20}$ Brenner, D. W., "Empirical Potential for Hydrocarbons for Use in Simulating the Chemical Vapor Deposition of Diamond Films," Physical Review B, Vol. 42, No. 15, Nov. 1990, pp. 9458-9471.

${ }^{21}$ Los, J. H. and Fasolino, A., "Intrinsic Long-Range Bond-Order Potential for Carbon: Performance in Monte Carlo Simulations of Graphitization," Physical Review B, Vol. 68, 2003, pp. 024107.

${ }^{22}$ Kalyanasundaram, N., Moore, M. C., Freund, J. B., and Johnson, H. T., "Stress Evolution Due to Medium-Energy Ion Bombardment of Silicon," Acta Materialia, Vol. 54, 2006, pp. 483-491.

${ }^{23}$ Humphrey, W., Dalke, A., and Schulten, K., "VMD - Visual Molecular Dynamics," Journal of Molecular Graphics, Vol. 14, No. 1, 1996, pp. 33-38.

${ }^{24}$ Berendsen, H. J. C., Postma, J. P. M., van Gunsteren, W. F., DiNola, A., and Haak, J. R., "Molecular Dynamics with Coupling to an External Bath," Journal of Chemical Physics, Vol. 81, No. 8, Oct. 1984, pp. 3684-3690.

${ }^{25}$ Sun, Q. and Boyd, I. D., "Evaluation of Macroscopic Properties in the Direct Simulation Monte Carlo Method," Journal of Thermophysics and Heat Transfer, Vol. 19, No. 3, 2005, pp. 329-335.

${ }^{26}$ Britton, M., Waters, D., Messer, R., Sechkar, E., and Banks, B., "Sputtering Erosion Measurement on Boron Nitride as a Hall Thruster Material," Tech. rep., NASA, 2002, NASA TM-2002-211837.

${ }^{27}$ Wilhelm, H. E., "Quantum-Statistical Analysis of Low Energy Sputtering," Australian Journal of Physics, Vol. 38, 1985, pp. 125-133. 